\title{
Pharmacogenomics in psychiatry: genomic considerations
}

\author{
Molecular Psychiatry (2005) 10, 713. doi:10.1038/ \\ sj.mp.4001721
}

In our previous editorial, we commented on the clinical aspects of pharmacogenomic research. We now focus on the genomic side. Once carefully phenotyped drug-response samples are collected, what genomic approaches should be used? This is an important open question in the field.

There are multiple issues to be addressed, going from the general and conceptual to the specific and technical. The first of those is whether to use a genome-wide, hypotheses-free approach, or to follow a candidate strategy. Going genome-wide is probably the way of the future, but for those doing research today, issues of feasibility and cost are still paramount. Completion of the International Human Haplotype Mapping Project has a strong likelihood of greatly reducing the cost of genome-wide approaches, provided that the haplotype strategy turns out to be really applicable and effective across the genome.

The alternative then is to use candidate approaches. How can those be pursued? One single-nucleotide polymorphism (SNP) at a time, one haplotype at a time, one gene at a time, a few genes at a time, an entire biological pathway at a time? As the cost of genotyping decreases it becomes increasingly hard to defend one SNP approach, in the absence of very compelling reasons. Those might be identification of a gene with a key functional SNP through linkage studies. Alternatively, a gene with an important variation may be the target for the drug in question, or a key downstream component of the drug response pathway. Additional considerations that are critical when SNPs and haplotypes are considered for study include their frequencies in a specific population and importantly in the group that's being studied.

At this crucial juncture should one still attempt to discover new SNPs in candidate genes or leave that approach in the hands of large, high-throughput initiatives, such as the Human Exon Project, which will resequence all 300000 human exons in 48 individuals? What about resequencing in ethnically identified populations for the purpose of discovering new SNPs or identifying SNP frequencies in specific populations?

A worthwhile endeavor is to identify genes associated with drug response as potential candidates for pharmacogenetic associations. This can broaden our search from a narrow list of expected candidates and might yield novel clues without the cost and complexity of genome-wide searches.

As one embarks on a candidate approach, myriad questions can be raised. Should SNPs or haplotypes of interest be characterized functionally? Should one systematically test as many SNPs and haplotypes as possible in a few, key genes, or should one cast a wider net, examining fewer SNPs, hopefully enriched for haplotype tags in many genes along critical biological pathways. Should priority be given to those variations affecting function?

Finally, novel statistical approaches are of fundamental relevance. How does one understand the significance of variables that cause small effects in the context of multiple testing in sample sizes that are limited by necessity? It is not possible to treat thousands of people, prospectively in standardized conditions, for long periods of time, as required for pharmacogenetics research. Therefore, statistics should determine levels of significance when limited samples are tested multiple times.

These topical issues also impact on what we can publish. Is it appropriate for us to consider for publication work that looks at small number of variations in limited samples? We believe it would not be helpful to set up strict criteria. Sometimes a small replication study in a different population can be of great value in assessing the validity of a novel candidate. Sometimes a larger study in which many candidates are looked at may not contribute much. Our approach has been to stay at the cutting edge of the field and to publish work that expands the frontier of existing knowledge. Those frontiers are being pushed fast; therefore, authors are encouraged to send presubmission letters of inquiry directly to us at molecularpsychiatry@mednet.ucla.edu. It is exciting to see the field move so rapidly. Only one thing is certain: whatever genomic approaches we use today will rapidly be surpassed. Constant evolution of one's approach is necessary. By the time genotyping is ready to be conducted, the approaches designed at the outset of a study may already be obsolete and reconsideration of strategies is highly recommended.

J Licinio and M-L Wong Center for Pharmacogenomics and Clinical Pharmacology, Semel Institute and David Geffen School of Medicine at UCLA

E-mail: licinio@ucla.edu 